photons discussed in our paper. It will be seen that the values given by the latter are strikingly supported by the experimental results.

C. V. Raman.

S. BHAGAVANTAM.

210 Bowbazar Street, Calcutta, India,

Nov, 29.

${ }^{1}$ Ind. Jour. Phy., vol. 6, p. 353, Oct. 1931

NATURe, 128 , pp. 576 and $727 ; 1931$.

\section{Wave-length of the Green Auroral Line Determined by the Interferometer.}

Some years ago Babcock ${ }^{1}$ gave results of accurate measurements with an interferometer method of the green line which appears in the light from the night sky. We have reason to believe that this line is identical with the strong green line appearing in the auroral spectrum; but on account of the great interest attached to this line it will be of importance to measure accurately the wave-length of the green line from the aurora itself in order to settle the question as to the identity of the two lines.

As I mentioned in a report at the Congress of the Geophysical Union at Stockholm, I was preparing for such investigations to be carried out at the Northlight Observatory at Tromsø, recently built by grants from the International Education Board founded by Rockefeller.

The apparatus used consists of a Fabry-Perot etalon placed in front of the lens of a camera. Two silvercoated quartz etalons-about 2.5 and $5 \mathrm{~mm}$. thick-

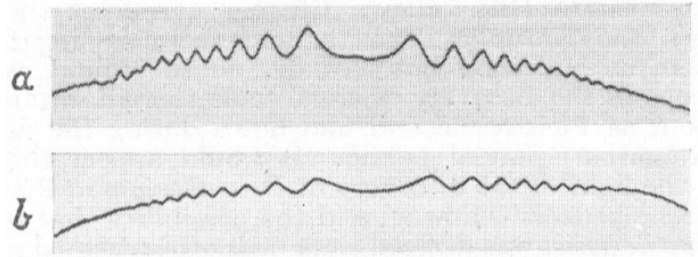

FIG. 1.

were made by Messrs. Adam Hilger, Ltd., London. Their optical thickness was measured by the National Physical Laboratory. The camera lens was an Ereman anastigmat of focal distance $10.5 \mathrm{~cm}$. and of high light power $F, 1: 1 \cdot 8$. The part of the light having a wave-length shorter than about $5200 \mathrm{~A}$. was removed by a Wratten filter placed in front of the etalon. The whole apparatus was put into an isolated box, where the temperature could be measured and kept fairly constant.

Recently two exposures of interference fringes of the auroral line were made with the $2.5 \mathrm{~mm}$. etalon at the Tromsø Observatory, one on Sept. 14 and the second on Oct. 29. Both exposures showed a large number of fringes (on the original plate 18-20 could be counted), and no sign of doubling or dissymmetry of the line was to be seen. The neon line $5852.488 \mathrm{~A}$. was used for comparison.

During the first exposures the regulation and measurements of the temperature were not satisfactory. During the exposures of Oct. 29, however, the temperature in the box on both sides of the etalon was measured at regular intervals.

Diagrams of the interference fringes corresponding to the exposures of Oct. 29 and obtained with a Moll microphotometer are shown in Fig. 1. The curves $(a)$ and $(b)$ correspond to the auroral line and the neon line respectively.

Details regarding standardisation of the plates and methods of calculation will be given in a subsequent paper. I will state here merely the results from the observations of Oct. 29, which may be considered as satisfactory.

The wave-length was calculated in the following two ways :

(1) The vacuum wave-length is found from the equation

$$
\lambda_{N}=\frac{2 e_{\mu}}{P_{N}^{-}}
$$

where $\mu$ is the refractive index and $e$ the thickness of the etalon reduced to the temperature of observation, $P_{N}$ is the order number for the centre of the auroral line ring-system. The true temperature was found by means of the neon line fringes.

(2) The comparison line was used directly and the wave-length found by means of the equation.

$$
\lambda_{N}=\frac{P_{C}^{\mu} \mu_{N V}^{v}}{P_{N} \mu_{C}} \lambda_{c}(1+\epsilon) \text {. }
$$

where $P_{c}, \mu_{C}, \lambda_{C}$ are order number, refractive index, and wave-length corresponding to the comparison line, $\epsilon$ is a small correction due to difference of temperature of the etalon at the exposure of the auroral line and that of the neon line. This temperature difference was measured directly.

Calculation according to (1) gave for the wavelength of the auroral line

$$
\lambda=5577 \cdot 340 \text { A. (I.U.). }
$$

The second method gave

$$
\lambda=5577 \cdot 345 \text { A. (I.U.). }
$$

The main error in our experiments is due to uncertainty in the temperature measurements, but should not exceed $0.01 \mathrm{~A}$. We are improving our arrangements so as to obtain a higher constancy of temperature.

As an average of his measurements, Babcock found $\lambda=5577 \cdot 350$. Thus, within the limit of error, the green line of the aurora is identical with that of the night sky luminescence.

$$
\begin{aligned}
& \text { Oslo, Nov. } 17 . \\
& { }^{2} \text { Astrophys. Jour., } 57 ; 1923 .
\end{aligned}
$$

\section{VEGARD,}

\section{Some Effects of Turbulent Pressure.}

IN a recent paper Ertel ${ }^{1}$ has shown, at some length, that turbulent motion in the atmosphere reduces the pressure, recorded by a barometer, by $\rho w^{\prime 2}$ dynes/ cm. ${ }^{2}$, where is the density of the air and $w^{\prime}$ is the vertical component of the turbulent velocity. This result we can get quite simply from analogy with the kinetic theory of gases, as pointed out by McCrea.2 Ertel estimates that a mean wind-speed near the ground of $10 \mathrm{~m}$./sec. would lower the local pressure by $0.08 \mathrm{mb}$.; a calculation from the observations of Scrase ${ }^{3}$ gives results of the same order.

It seems unnecessary that there should be equi. partition of eddying energy in the three directions $O x$ down-wind, $O y$ across, and $O z$ vertical; this, in fact, is shown from the observations of Scrase on pages 6 and 12 of the paper quoted. Considering in a general way the manner of formation of the eddies by friction at the earth's surface, one would suppose that the $u^{\prime}$ and $w^{\prime}$ components of the eddying motion would be of larger order than the $v^{\prime}$ (cross-wind) component, especially in observations taken over a short period, like one minute; but Scrase shows that the $v^{\prime}$ component is $30-50$ per cent greater than the $w^{\prime}$, particularly near the ground ( 1.5 metres).

Now consider two points $P$ and $Q$ at the same level and at, for example, 100 metres from one another across wind; then the surface 'roughness' at $P$ and

No. 3244, VoL. 129] 